\title{
Representations of odor plume flux are accentuated deep within the moth brain
}

Thomas C Baker

Address: Center for Chemical Ecology, Department of Entomology, 105 Chemical Ecology Laboratory, Penn State University, University Park, PA 16802, USA. Email: tcb10@psu.edu

Published: 20 February 2009

Journal of Biology 2009, 8:16

The electronic version of this article is the complete one and can be found online at http://jbiol.com/content/8/2/16

(c) 2009 BioMed Central Ltd

\begin{abstract}
Odor space, the representation of odor quality in the insect brain, is known to be optimally resolved when lateral inhibitory pathways are functioning normally. A new study published in the Journal of Biology now shows that odor time resolution also depends on the normal functioning of such pathways.
\end{abstract}

\section{Odor space and odor time}

Understanding how insects detect, discriminate, and act upon relevant olfactory stimuli such as pheromones and host odors has been a major challenge for researchers for decades. The 'act upon' part of this challenge involves understanding insects' odor- mediated behavior; that is, how they maneuver when they smell something relevant. Much has been learned over the years about sexpheromone-mediated flight maneuvers in moths, and much has also been learned about odor discrimination from work on moth sex pheromone systems. The olfactory part of these systems involves the activities of an array of thousands of tightly and differentially tuned olfactory receptor neurons (ORNs) on the male antenna, imbuing it with a distributed specificity of signal acquisition for each of the two or three sex pheromone components in the blend. Acquisition is followed by signal processing by networks of interneurons that form a fine-grained odor quality pattern-recognition system. One part of sex pheromone olfaction thus involves a sampling and reporting of the relative abundances of the different chemicals that comprise the blend and classifying the resulting pattern of neuronal excitation as occupying a certain behaviorally effective position in 'odor space' [1].
Another, less investigated aspect of pheromone olfaction involves temporal odor resolution, and in a new paper in the Journal of Biology Lei et al. [2] present findings that illuminate new features of the temporal fine tuning that goes on in moths' pheromone olfactory pathways. Notably, in a rare effort they directly and elegantly link the impairment of inhibitory circuits in the signal-processing network in the moth's antennal lobe with behavioral impairment of upwind flight.

Lei et al. point out that the task for the insect's olfactory system "is to resolve the spatiotemporal dynamics of olfactory stimuli" in an odor plume, and they have focused on the temporal portion. A pheromone odor plume can be envisioned as having been sheared from its emission source as a strong single strand. The strand is then stretched and shredded into myriad sub-strands by turbulence [3] as it is transported by larger-scale turbulent air masses away from the source along fairly straight lines out into the environment. Because insects' olfactory receptor organs, their sensilla, are directly exposed to wind flowing over them, they are subjected to an odor flux from odor strands and the clean air pockets between strands that usually varies over milliseconds. 
The pheromone blend occurs in every strand, and moths discriminate and behaviorally respond to it on a single strand basis, resulting in optimal maneuvering for upwind flight within a couple of hundred milliseconds of a signal being received [4]. The spatial position of the time-averaged plume or its strands relative to the environment has not been shown to be sensed by the olfactory system. So for research on the spatiotemporal dynamics of olfaction, the 'spatio' portion might be viewed as the brain's representation of a particular blend in odor space, not in environmental space.

\section{Moths follow the wind when their nose tells them to}

Flying male moths responding to pheromone do not steer according to the chemical concentration in environmental space. In other words, they do not 'follow their nose'; they follow the wind when their nose tells them to. In insects there is a need for speed in olfaction [4], and the ORNs are built to be flux detectors rather than concentration analyzers. The key element of ORNs that allows flux detection is a self-cleaning feature provided by the pheromone-binding proteins and degradative enzymes bathing an ORN. Within milliseconds, this gets rid of lingering pheromone molecules after each strand contact and allows the ORNs to disadapt and be able to respond with high fidelity to the next strand.

Lei et al. have now shown that further downstream, in postsynaptic olfactory pathways, inhibitory $\mathrm{GABA}_{\mathrm{A}}$-ergic interneurons act to clean up the action-potential activity lingering between strand-induced bursts. These neurons reduce inter-strand action-potential frequency and preserve in the brain a high-fidelity representation of the environmental odor flux that is being reported by the ORNs. The odor-flux peaks of the plume's pheromone strands and the troughs of the clean air pockets are sharpened by the antennal lobe's inhibitory circuitry, and their temporal integrity is kept intact deep within the olfactory system.

\section{Sharpened olfactory temporal resolution related to high-speed flight maneuvers}

The challenge for insects is to sample the odor strands as frequently as possible, as well as to sample the inter-strand pockets to make sub-second, in-flight decisions about maneuvering in the wind flow, whose direction of movement provides the moth with the only information available about the toward-source direction. Failure to perform these feats of olfactory temporal acuity that, as Lei et al. [2] have shown, are linked to proper wind-steering maneuvers, can mean failure to find a mate before competitors do. Not responding rapidly enough to contact with a strand can result in lack of progress straight upwind to the source. Failure to rapidly respond to a pocket of clean air between strands by not immediately stopping upwind progress and initiating side-to-side crosswind 'casting' flight can result in erroneous steering, both off-line from the toward-source direction as well as away from the direction to which the plume has swung in a shifting wind field [5].

The insect signal acquisition and processing system for sex pheromone starts with neuronal inputs from the tens of thousands of ORNs on the male antenna, each ORN being differentially and tightly tuned to only one of the two or three components that comprise that species' blend of sex pheromones. Axons from each of these classes of pheromone-component-tuned ORNs travel to the antennal lobe of the brain at the base of the antenna and arborize in their own class-specific knot of neuropil called a glomerulus, which resides there within a cluster of other pheromone-component-specific glomeruli called the macroglomerular complex (MGC; Figure 1). It is here that the first postsynaptic interneurons, called local interneurons, impose GABA-related inhibition on neurons in neighboring MGC glomeruli.

This form of olfactory lateral inhibition has been implicated in enhancing the contrast between the activities across the ensemble of glomeruli to produce a contrast-enhanced relative pattern of outputs across the array of different projection interneurons exiting the various glomeruli and projecting out to the mushroom bodies and the lateral protocerebrum (Figure 2). The across-ensemble pattern of projection interneuron activity results in a representation of pheromone blend quality as a spatial pattern in the mushroom body. An earlier study [6] demonstrated the effects of a GABA blocker, picrotoxin, on odor-space discrimination. Impairing the activities of GABA-ergic neurons and dampening local field potential oscillations (believed to be set up by interactions between the antennal lobe and mushroom bodies) reduced fine-grained odor-quality discrimination by honey bees.

Lei et al. have now demonstrated the importance of lateral inhibition in the temporal domain of olfactory acuity. They used bicuculline methiodide to block the activity of $\mathrm{GABA}_{\mathrm{A}}$ inhibitory pathways in the pheromone-related glomeruli of the moth MGC and showed that these pathways work to silence neuronal firing of projection interneurons in the clean-air pockets between pheromone strands. Impairing the GABA-ergic neurons did not affect peak firing in response to pheromone strands, so the significant reduction in projection neuron firing between strand-induced bursts helps improve temporal resolution and accentuate the variations in pheromone flux. 


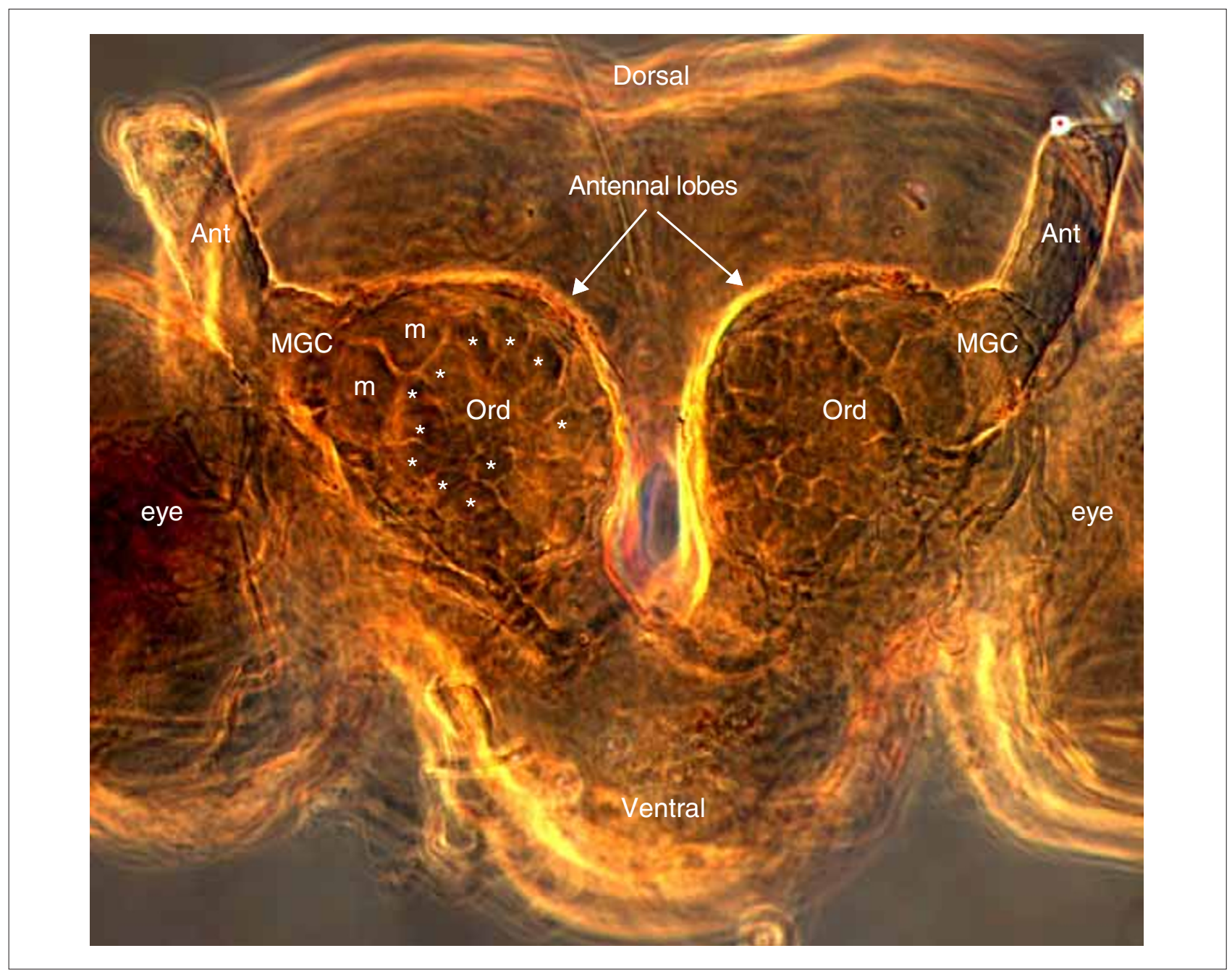

Figure I

Frontal view of the face of a male Helicoverpa zea moth showing the two antennal lobes at the bases of the antennae. The preparation has been histologically cleared so that the many antennal lobe glomeruli are visible as spheroidal shapes. Asterisks denote 'ordinary' glomeruli that receive inputs from antennal neurons responding to general environmental odorants such as plant volatiles. The ordinary glomeruli reside in a large cluster in each antennal lobe (Ord). Larger glomeruli that receive inputs from pheromone component-tuned neurons on the antenna reside in their own cluster called the macroglomerular complex (MGC) and are labeled with an ' $m$ '. Ant, the remaining bases of the antennae and antennal nerves; eye, optic lobe.

Notably, Lei et al. directly linked impairment of the temporal contrast-enhancement circuitry in the antennal lobe with impaired upwind flight behavior of male moths. They thus demonstrated the importance of temporal pheromone strand resolution by the inhibitory antennal lobe circuits to successful pheromone source location by flying moths. Researchers decades earlier had demonstrated the importance of pheromone flux variations to successful upwind flight behavior by manipulating the pheromone plume flux itself and not the olfactory pathways, as Lei et al. have done in their current study.
After RH Wright [7] first pointed out that odor plumes are composed of small strands of highly concentrated odor that might be important in influencing insect behavior, subsequent studies showed that flux change, that is, pheromone intermittency, is crucial for successful upwind flight by males. Presentation of otherwise attractive pheromone odors as a uniform fog or cloud caused no upwind flight, just side-to-side cross-wind casting flight [8]. When such clouds were pulsed and interspersed with clean air at a frequency of 1 or $2 \mathrm{~Hz}$, upwind flight proceeded successfully [9]. Further experiments suggested that individual 


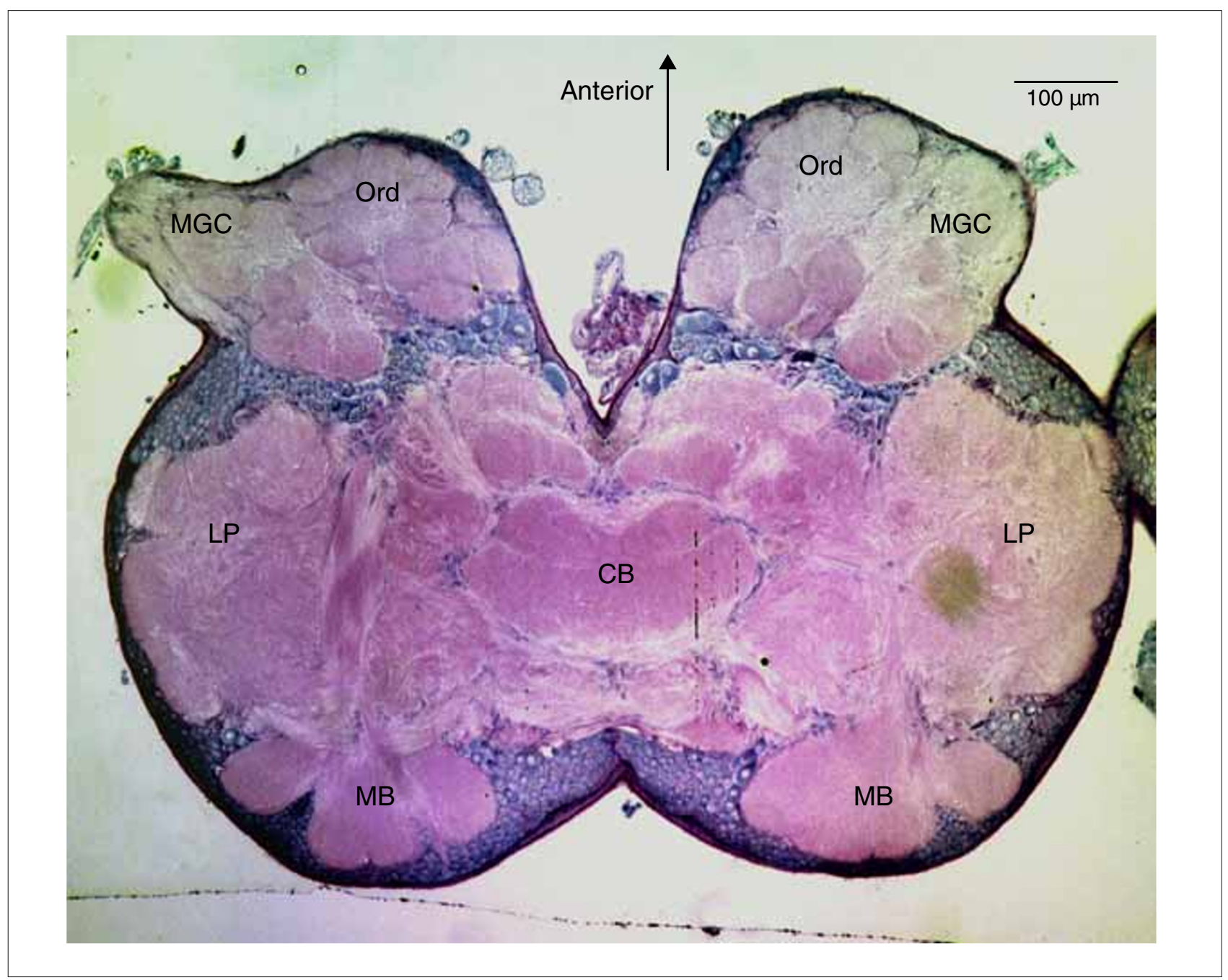

\section{Figure 2}

Top view of the head of a Helicoverpa zea male moth stained histologically to highlight the regions of the male moth brain involved with pheromone and other odorant signal processing and odor-quality discrimination. The anterior face of the moth is looking up toward the top of the figure. Sex pheromone information comes into the antennal lobe glomeruli of the macroglomerular complex (MGC) from the antenna. General odorant information comes from the antenna into the ordinary glomeruli (Ord) of the antennal lobe. Inhibitory GABA-ergic local interneurons form a network cross-linking all the antennal lobe glomeruli and help shape the relative levels of excitation emerging from each glomerulus via projection interneurons. The axons of these projection interneurons project in a single tract to the back of the brain to synapse first with neuropil in the mushroom body (MB) before continuing on to synapse with neurons in the lateral protocerebrum (LP). Axons of other projection neurons that also carry relative levels of excitation from antennal lobe glomeruli project in a second, different tract directly to the LP, bypassing the MB. The LP is where behavior-initiating descending interneurons synapse to send command signals to motor centers. Adapted from Lee et al. [I I].

strands within a plume could evoke upwind flight behavior, and experimentally generated single strands were shown to promote single upwind flight 'surges' within approximately 0.3 seconds after strand contact (see $[5,10]$ and references therein). Equally fast reaction times to pockets of clean air were suggested to be as behaviorally important for successful and rapid source location as the reaction to the strands themselves; hence, the selection over evolutionary time for high-fidelity flux resolution in moth pheromone olfactory systems [5].

Lei et al. have convincingly demonstrated the importance of inhibitory $\mathrm{GABA}_{\mathrm{A}}$-ergic circuitry in preserving a high-fidelity temporal representation of pheromone flux in projection interneurons deep within moths' pheromone olfactory pathways. Previously known to be important for optimizing 
odor quality discrimination, GABA-ergic interneurons have now been shown to be behaviorally important enhancers of temporal olfactory acuity. Some types of projection interneurons arborize first in the mushroom body and then in the lateral protocerebrum (Figure 2), where synapses with behavior-generating descending interneurons occur. Another type projects directly to the lateral protocerebrum, bypassing the mushroom body. It seems possible that because there are two distinct odor-resolution systems in insect olfaction, one for high-fidelity representation of odor space and another for high-fidelity reporting of odor time, moths may use these two different pathways in the brain that have been selected over evolutionary time for different, but complementary, behavioral purposes.

\section{Acknowledgements}

I thank Neil Vickers for reading through a penultimate draft of this paper and providing many helpful comments.

\section{References}

I. Hallem EA, Carlson JR: Coding of odors by a receptor repertoire. Cell 2006, I25:143-160.
2. Lei H, Riffell JA, Gage SL, Hildebrand JG: Contrast enhancement of stimulus intermittency in a primary olfactory network and its behavioral significance. J Biol 2009, 8:21.

3. Murlis J: The structure of odor plumes. In Mechanisms in Insect Olfaction. Edited by Payne TL, Kennedy CEJ, Birch MC. Oxford: Clarendon Press; 1986:27-39.

4. DeBruyne M, Baker TC: Odor detection in insects: Volatile codes. J Chem Ecol 2008, 34:882-897.

5. Baker TC, Vickers NJ: Pheromone-mediated flight in moths. In Pheromone Research: New Directions. Edited by Cardé RT, Minks AK. New York: Chapman and Hall; 1997:248-264.

6. Stopfer M, Bhagavan S, Smith BH, Laurent G: Impaired odour discrimination on desynchronization of odour-encoding neural assemblies. Nature 1997, 390:70-74.

7. Wright RH: The olfactory guidance of flying insects. Can Entomol 1958, 90:8I-89.

8. Kennedy JS: Zigzagging and casting as a programmed response to wind-borne odour: a review. Physiol Entomol 1983, 8:109-120.

9. Baker TC, Willis MA, Haynes KF, Phelan PL: A pulsed cloud of sex pheromone elicits upwind flight in male moths. Physiol Entomol 1985, 10:257-265.

10. Vickers NJ, Baker TC: Reiterative responses to single strands of odor promote sustained upwind flight and odor source location by moths. Proc Natl Acad Sci USA 1994, 91:5756-5760.

II. Lee SG, Carlsson MA, Hansson BS, Todd JL and Baker TC: Antennal lobe projection destinations of Helicoverpa zea male olfactory receptor neurons responsive to heliothine sex pheromone components. J Comp Physiol A 2006, 192:35I-363. 